\title{
Survey on Removal of Universal Noise from Images
}

\author{
Muthuselvi S \\ Post Graduate Scholar \\ Department of information technology \\ Karunya University \\ Coimbatore, India
}

\author{
Narmadha D \\ Lecturer \\ Department of information technology \\ Karunya University \\ Coimbatore, India
}

\begin{abstract}
Removing mixed noise from images is one of the challenging problem. In this paper, review the types of filtering approach and noise detection technique for remove mixed noise from images. Switching bilateral filter is one of the best methods for removing mixed noise. Compare to other filters, the switching scheme is best one for restore high level impulse noise in images. Computational complexity of SBF is less than the other universal noise removal filters.
\end{abstract}

\section{General Terms}

Image Denoising, Universal noise, MATLAB.

\section{Keywords}

Gaussian noise, Impulse noise, Mixed noise, Noise detection, Impulse noise, Switching bilateral filter

\section{INTRODUCTION}

The essential image processing task is an image denoising, to reduce noise from images. Noise can be methodically familiarized into images throughout acquisition and transmission. The most important problem of image processing is to successfully remove noise from an image at the same time as keeping its features secure. Noise exclusion is tricky task because images may be corrupted by different types of noise, such as additive, impulse or signal dependent noise. Using linear filter to remove additive gaussian noise from images. Though, linear filtering technique blur edge details in images and it fails to remove impulse noise. Avoid this problem nonlinear filter technique have to be used. In this paper, describe SBF filtering technique for both gaussian and impulse noise removal. both gaussian and impulse noise removal. Gaussian noise is characterized by adding to each image pixel a value with a zero-mean Gaussian distribution [2], [5].Such noise is usually introduced during image acquisition.The zero-mean distribution property allows such noise to be removed by averaging pixel values[5]. Impulse noise is characterized by replacing a portion of an image pixels with noise values, the remaining pixels be unchanged [13]. SBF achieves high peak signal to noise ratio and great image quality. In section II describe the methodology for universal noise removal. Section III gives a brief description of the analysis of various noise removal techniques and algorithm. Section IV gives the comparative analysis of SBF and other noise removing filter.

\section{METHODOLOGY FOR UNIVERSAL NOISE REMOVAL}

Many types of algorithms is provided to filter the images from noise. In this survey paper we analyze various algorithms to remove both impulse noise and gaussian noise. Median filter, mainly used to removing impulse noise, it provide best performance of removing impulse noise from corrupted images by replacing the noisy pixel through the median value of the pixel from a selected neighborhood. The SDROM filter suggest by Abreu in 1996, this filter capability to remove gaussian noise in addition to mixed gaussian and impulse noise[14]. Center weight median filter was proposed by Ko and Lee to remove impulse noise from images. This center weight median filtering technique gives additional weight merely to the central pixel value of a window. More amount of image details are preserved, but blurring effect is still experiential. Peng and Lucke recommended a fuzzy filter planned particularly for mixed noise removal [9]. In 1979 bilateral filtering was first introduced by Overton and Weymouth [4]. It's a preprocessing tool for removing noise. In 1998, Tomasi and Manduchi [4] provided the name bilateral filtering and enhanced the method in several aspects.

Elad [10] established a connection between bilateral filtering and several other methods in terms of minimize functional. Roman Garnett, et al., was proposed trilateral filter for removing both gaussian and impulse noise from images. Local image statistic intended for find out noise pixels in images. The directional weight median filter was suggest by Yiqiu Dong, et al., to remove impulse noise from images. Directed weight median filter uses an iterative approach. This filter restrains impulses by means of high noise level. Zhou wang, et al., proposed by progressive switch median filter. In this filter restore images ruined by salt and pepper impulse noise. The switching scheme, impulse detection algorithm is used to detect the noise then filtering is applied to corrupted pixels. Noise detection and filtering actions are increasingly applied through several iterations. Chih-Hsing Lin, et al., was Proposed switching bilateral filter for universal noise removal. From the above noise removal filtering techniques, few are selectively analyzed in detail in this literature.

\subsection{Generalization of Median Filtering Using Order Statistic}

Median Filter is a uncomplicated and potent non-linear filter. This filter based upon order statistics and simple to execute method of smooth images, that is Plummeting the amount of intensity variation among one pixel and the next. It is frequently used to reduce noise in images. The median filtering is through by arrange ascending order all the pixel value in surrounding neighborhood then replace every pixel value with the median value of pixels in the window. If $\mathrm{V}(\mathrm{i}, \mathrm{j})$ and $\tilde{V}(i, j)$ be the input and output respectively, the median filter is defined as follows $\tilde{V}(i, j)=$ median $\{\mathrm{V}(\mathrm{i}-\mathrm{s}, \mathrm{j}-\mathrm{t}) \mid(\mathrm{s}, \mathrm{t}) \in$ $\mathrm{W}$ ) Where $\mathrm{W}$ is the window that is define in terms of the image coordinates in the neighborhood of the origin. 


\begin{tabular}{|l|l|l|l|l|}
\hline & & & & \\
\hline & 22 & 6 & 23 & \\
\hline & 12 & 90 & 15 & \\
\hline & 9 & 5 & 16 & \\
\hline & & & & \\
\hline
\end{tabular}

Fig 1: Method of median filtering

Neighbourhood Values: 569121516222390 .

Median value: 15 .

In Fig 1 shows the center pixel value of 90 in the $3 \times 3$ window, the center pixel value is replaced by median value of 15.

\subsection{Directional Weight Median Filter for Removal of Impulse Noise}

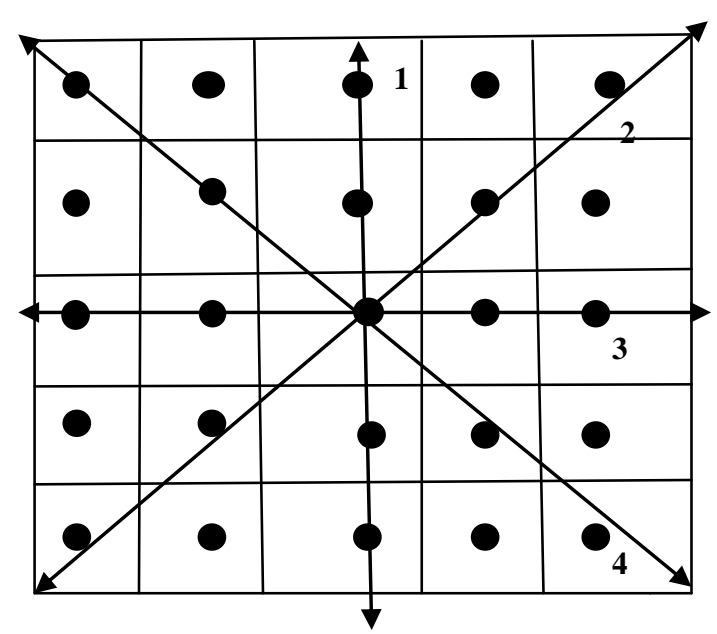

Fig 2: Directional method for impulse detection

To remove random valued impulse noise from images using directional weight median filter. In Fig 2 Impulse to detect the noisy pixel in an image, which is based upon distinction between current pixel and surrounding with four directions [3]. This filter using an iterative filtering approach. Weighted differences are calculated during each direction and take minimum value. Compared a minimum value with threshold value, which is greater than threshold value the pixel is considered as noisy pixel [3]. The noisy pixels values are do not put back by output of the median filter, keep on to use information of four directions to weight the pixel in the window to preserve detail as removing noise. By an adaptive weighted median filter evaluation is done. This filter gives better performance of suppress high noise level.

\subsection{Bilateral Filtering for Gray and Color Images}

Bilateral filter is a nonlinear technique that be able to blur an image at the same time as respect to strong edges. The bilateral filter have good results in removing noise at the same time as preserving edges in images [4], [16]. This method is noniterative, local and simple [4], [16]. Bilateral filtering is an efficient way to smooth an image as preserving its discontinuities. Bilateral filter don't sufficiently remove impulse noise for the reason that the noise pixel and surrounding pixels difference are too large. Consequently, local weighting is also small to change the noise value. Designed the weighting function in the direction of even in regions of similar intensity at the same time as keeping edges intact, through heavily weighting those pixels that are both close to the central pixel spatially and similar to the central pixel radiometrically. os and or parameters are control the behavior of weight.

\subsection{Universal Noise Removal Algorithm with an Impulse Detector}

The trilateral filter is a alteration of the bilateral filter, with ROAD statistic incorporated into a bilateral filter for impulse noise detection [4], [2]. In this filter carry out well in removing both gaussian and impulse noise from images.

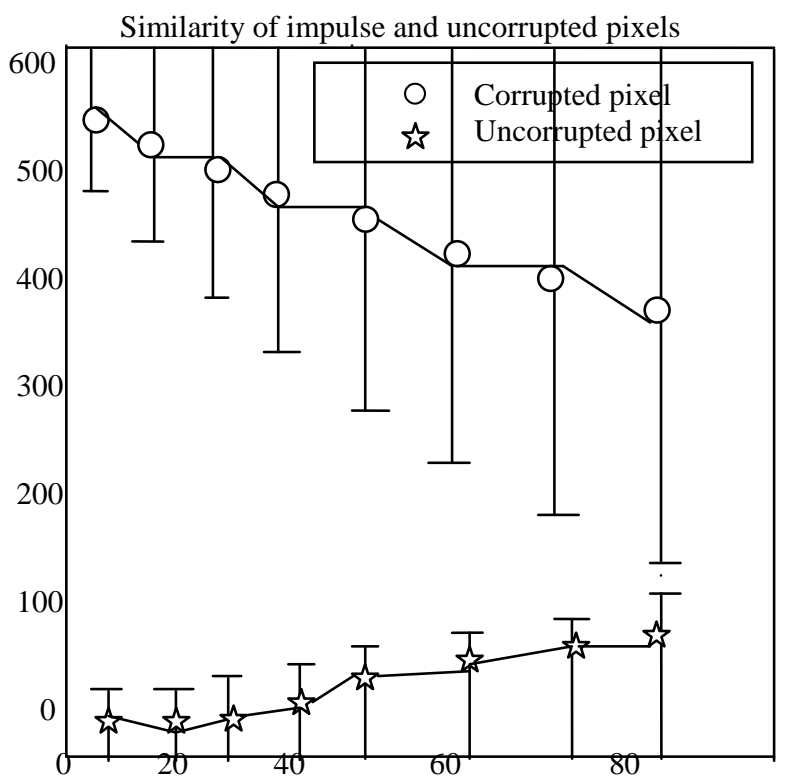

Possibility of impulse noise

Fig 3: Calculate ROAD value

Fig 3 ROAD statistic is a best estimate of impulse noise. Statistic to be very high for noisy pixels and lower for uncorrupted pixels. The trilateral filter can restore corrupted images with small to modest levels of impulse noise with practically unblemished results. Trilateral filter can remove a fair amount of noise even as preserve edge boundaries and well details. Restoration performances are measured by peak signal to noise ratio.

\subsection{Switching Bilateral Filter with a} Texture and Noise Detector for Universal Noise Removal

Most often perform image processing operation is noise removal, while the enhancement of images. There are many noise and edge detectors with specific algorithm are available for removing noise. Detection are based upon robust Estimators and followed by filtering. Impulse detection and classification rate, shows a good performance in identifying noise even in mixed noise models. The switching scheme, noise detector to identify noisy pixels and differentiate the 
noisy pixels from noise free pixels.In this paper we propose universal noise removal filter and SQMV.

This filter is used to remove mixed noise, salt and pepper with uniform noise and salt and pepper with Gaussian noise. Sorted quadrant median vector is used to recognize edge and texture. Difference between the current pixel and reference medians are calculated for detect noise. If differences are large, the pixel is considered as noisy pixel. Alternatively, when the differences are small, the pixel is considered as noise free pixel. Non proper reference median would guide to incorrect detection. So we have to find proper reference median. In Proper reference median can be selected depend upon edge and texture information.

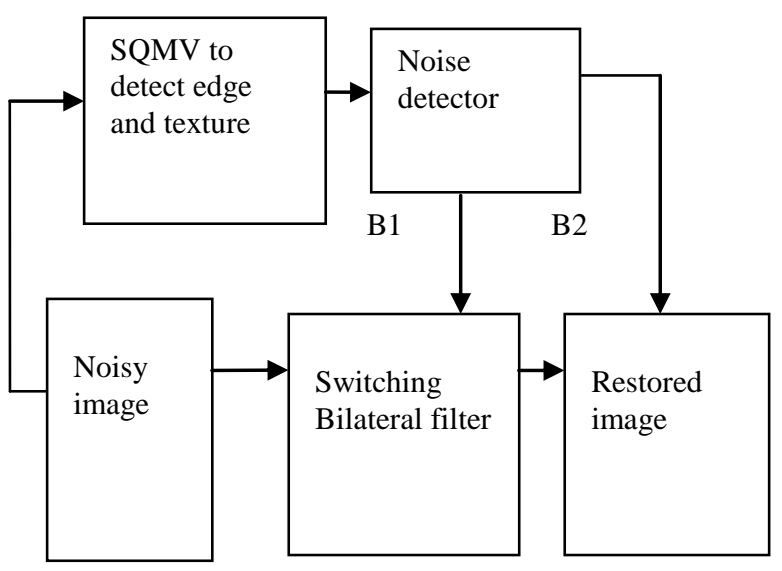

Fig 4: Switching scheme

In Fig.4. detection of noise is completed in two stages. The reference median is choosing for noise detection in an image. The noise detector in addition decides whether a current pixel should be filtered through an SBF or whether it should bypass the SBF. Switching bilateral filter is divided into two kinds, one is SBFim and another one is SBFga. In B1, B2 denote switching control signal created by the noise detector.The restored image has the original pixels if noise detector and edge and texture detector identify the pixels as noise free. If the pixel is detect impulse noise, then the pixel is processed by the SBFim. If the pixel is does not recognized as an impulse noise, although the current pixel and the reference median differences is still larger than a threshold, after that pixel is identified as Gaussian noise and is filtered by SBFga.

\section{ANALYSIS OF UNIVERSAL NOISE REMOVAL ALGORITHM AND TECHNIQUES}

The most important parameters considered for analysis of restoration performance are measured by peak signal to noise ratio, mean squared error, and mean absolute error, image enhancement filter. Restoration performances give the better image quality and details. Many types of algorithm and technique have been offered for restoration of images.

\subsection{Merits of Generalization of Median} Filtering Using Order Statistic

i) Median filter is very simple to implement.

ii) Median filter restore the brightness drops, object edges and local peaks in noisy images.

\subsection{Demerits of Generalization of Median Filtering Using Order Statistic}

i) Median filter removing the image details while reducing the noise.

ii) The median filter perform disappointing result when the signal weakening.

\subsection{Merits of Directional Weight Median Filter for Removal of Impulse Noise \\ i) Suppress impulse with high noise level. \\ ii) Preserve more detail, even thin lines. \\ iii) Restore color images.}

\subsection{Demerits of Directional Weight Median Filter for Removal of Impulse Noise \\ i) Some noisy pixels are missed. \\ ii) Noise free pixel identified as noisy pixel.}

\subsection{Merits of Bilateral Filter For Gray and Color Images}

i) Bilateral filter is removing noise effectively while preserve edge details.

ii) Bilateral filter is noniterative, local and simple.

\subsection{Demerits of Bilateral Filter for Gray and Color Images}

i) Bilateral filter gives poor smoothing in high gradient regions.

ii) The bilateral filter cannot effectively remove impulse noise.

\subsection{Merits of Universal Noise Removal} Algorithm with an Impulse Detector i) Trilateral filter preserve edge boundaries.

ii) Eliminate more amount of noise.

iii) Best visual quality

3.8 Demerits of Universal Noise Removal Algorithm with an Impulse Detector

i) Leave less amount of noise in restored images.

ii) If half of the pixels in the image window are corrupted while ROAD value could be false.

3.9 Merits of Switching Bilateral Filter with a Texture and Noise Detector for Universal Noise Removal

i) Quality of image is best.

ii) Peak signal to noise ratio is high.

iii) Preserve edge details.

iv) Computational complexity is low.

\subsection{Demerit of Switching Bilateral Filter with a Texture and Noise Detector for Universal Noise Removal}

i) In the low level of impulse noise, the classification ratio is not good. 


\section{EXPERIMENTAL EVALUATION}

The proposed switching bilateral filter algorithm and impulse detection technique are compared with other algorithms and techniques for computing the results. In the noise corrupted image is passed through the edge and noise detector and the window size is $5 * 5$ then the window divides into four $3 * 3 \mathrm{sub}$ windows, by the center pixel is a corner pixel of four sub windows. In the noise detector, image pixels are processed row by row from top to bottom and, left to right from every row. Select the threshold for identifying impulse noise or gaussian noise.

TK1 $£\{35,60\}$, TK $2 £\{5,20,25\}$

The choice of $[\mathrm{TK} 1, \mathrm{TK} 2]=[60,25]$ produce acceptable results in filter salt and pepper noise, next selection of [TK1, TK2] $=[35,5]$ accomplish well for removing both Uniform impulse and gaussian noise. In the corrupted image, noisy pixel is first processed through edge and noise detector, then filtered by switching bilateral filter. Proposed technique produced best visual image quality and PSNR measures than other method.

Table 1. Comparison of proposed method with 2.1

\begin{tabular}{|c|c|c|}
\hline Parameters & $\mathbf{2 . 1}$ & $\begin{array}{c}\text { Proposed } \\
\text { approach }\end{array}$ \\
\hline Algorithm & $\begin{array}{c}\text { Median filtering } \\
\text { algorithm is used } \\
\text { to removing } \\
\text { impulse noise } \\
\text { from images. }\end{array}$ & $\begin{array}{c}\text { Switching bilateral } \\
\text { filter is used to } \\
\text { remove universal } \\
\text { noise from images. }\end{array}$ \\
\hline Technique & $\begin{array}{c}\text { Impulse detection } \\
\text { is used to detect } \\
\text { noisy pixel and } \\
\text { replace with } \\
\text { median value. }\end{array}$ & $\begin{array}{c}\text { Sorted Quadrant } \\
\text { median vector is } \\
\text { used for edge and } \\
\text { texture and noise } \\
\text { detection. }\end{array}$ \\
\hline $\begin{array}{c}\text { Performance } \\
\text { measured }\end{array}$ & $\begin{array}{c}\text { PSNR value is } \\
\text { low. }\end{array}$ & $\begin{array}{c}\text { PSNR value is high. } \\
\text { PSnd }\end{array}$ \\
\hline
\end{tabular}

Table 2. Comparison of proposed method with 2.2

\begin{tabular}{|c|c|c|}
\hline Parameters & $\mathbf{2 . 2}$ & $\begin{array}{c}\text { Proposed } \\
\text { approach }\end{array}$ \\
\hline Algorithm & $\begin{array}{c}\text { Directional weight } \\
\text { median filter for } \\
\text { removal of random } \\
\text { valued impulse } \\
\text { noise from images. }\end{array}$ & $\begin{array}{c}\text { Switching bilateral } \\
\text { filter is used to } \\
\text { remove universal } \\
\text { noise from images. }\end{array}$ \\
\hline
\end{tabular}

\begin{tabular}{|c|c|c|}
\hline Technique & $\begin{array}{c}\text { Iterative filtering } \\
\text { approach is used to } \\
\text { high accuracy of } \\
\text { detection. }\end{array}$ & $\begin{array}{c}\text { Sorted Quadrant } \\
\text { median vector is } \\
\text { used for edge and } \\
\text { texture and noise } \\
\text { detection. }\end{array}$ \\
\hline $\begin{array}{c}\text { Performance } \\
\text { measured }\end{array}$ & $\begin{array}{c}\text { PSNR value is } \\
\text { lower than } \\
\text { proposed method. }\end{array}$ & $\begin{array}{c}\text { PSNR value is } \\
\text { high. }\end{array}$ \\
\hline
\end{tabular}

Table 3. Comparison of proposed method with 2.3

\begin{tabular}{|c|c|c|}
\hline Parameters & $\mathbf{2 . 3}$ & $\begin{array}{c}\text { Proposed } \\
\text { approach }\end{array}$ \\
\hline Algorithm & $\begin{array}{c}\text { Removing gaussian noise } \\
\text { from images using } \\
\text { bilateral filter. }\end{array}$ & $\begin{array}{c}\text { Switching } \\
\text { bilateral filter is } \\
\text { used to remove } \\
\text { universal noise } \\
\text { from images. }\end{array}$ \\
\hline Technique & $\begin{array}{c}\text { Local measure is used for } \\
\text { detection. The noisy pixel } \\
\text { is replaced with a } \\
\text { weighted average of the } \\
\text { neighborhood } \\
\text { Intensities. }\end{array}$ & $\begin{array}{c}\text { Sorted } \\
\text { Quadrant } \\
\text { median vector } \\
\text { is used for edge } \\
\text { and texture and } \\
\text { noise detection. } \\
\text {. }\end{array}$ \\
\hline $\begin{array}{c}\text { Performance } \\
\text { measured }\end{array}$ & $\begin{array}{c}\text { PSNR value is lower than } \\
\text { proposed method. }\end{array}$ & $\begin{array}{c}\text { PSNR value is } \\
\text { high. }\end{array}$ \\
\hline
\end{tabular}

Table 4. Comparison of proposed method with 2.4

\begin{tabular}{|c|c|c|}
\hline Parameters & $\mathbf{2 . 4}$ & $\begin{array}{c}\text { Proposed } \\
\text { approach }\end{array}$ \\
\hline Algorithm & $\begin{array}{c}\text { To remove mixed } \\
\text { noise from images } \\
\text { using trilateral filter. }\end{array}$ & $\begin{array}{c}\text { Switching } \\
\text { bilateral filter is } \\
\text { used to remove } \\
\text { universal noise } \\
\text { from images. }\end{array}$ \\
\hline Technique & $\begin{array}{c}\text { ROAD statistic is } \\
\text { used to noise } \\
\text { detection. This } \\
\text { statistic to be very } \\
\text { high for noisy pixels. }\end{array}$ & $\begin{array}{c}\text { Sorted Quadrant } \\
\text { median vector is } \\
\text { used for edge and } \\
\text { texture and noise } \\
\text { detection . }\end{array}$ \\
\hline $\begin{array}{c}\text { Performance } \\
\text { measured }\end{array}$ & $\begin{array}{c}\text { PSNR value is lower } \\
\text { than proposed } \\
\text { method. }\end{array}$ & $\begin{array}{c}\text { PSNR value is } \\
\text { high. }\end{array}$ \\
\hline
\end{tabular}




\section{CONCLUSION}

In this paper present, new proposed method of switching bilateral filter and Sorted quadrant median vector for detect and removing universal noise from images. Survey for various noise removal algorithm and techniques was done. Comparative analysis with other recently proposed methods based on the PSNR and various techniques proves that the proposed method is superior in terms of image quality.

\section{ACKNOWLEDGMENTS}

The authors would like to thank ALMIGHTY GOD whose blessings have bestowed in me the will power and confidence to carry out my work.

\section{REFERENCES}

[1] A. C. Bovik, T. S. Huang, and D. C. Munson, "A generalization of median filtering using linear combinations of order statistics," IEEE Trans. ASSP, vol. 31, pp. 1342-1350, Dec. 1983.

[2] R. Garnett, T. Huegerich, C. Chui, and W. He, "A universal noise removal algorithm with an impulse detector," IEEE Trans. Image Process., vol. 14, no. 11, pp. 1747-1754, Nov. 2005.

[3] Y. Dong and S. Xu, "A new directional weighted median filter for removal of random-valued impulse noise," IEEE Signal Process. Lett., vol. 14, no. 3, pp. 193-196, Mar. 2007

[4] C. Tomasi and R. Manduchi, "Bilateral filtering for gray and color images," in Proc. IEEE Int. Conf. Computer Vision, 1998, pp. 839-846.

[5] W. K. Pratt, Digital Image Processing. New York: Wiley, 1978.

[6] S. J. Ko and Y. H. Lee, "Center weighted media filters and their applications to image enhancement," IEEE Trans. Circuits Syst., vol. 38, no. 9, pp. 984-993, Sep. 1991.

[7] R. C. Hardie and K. E. Barner, "Rank conditioned rank selection filters for signal restoration," IEEE Trans.Image Process., vol. 3, no. 3, pp. 192-206, Mar. 1994.
[8] Ms. Neha Jain, "Noise cancellation using Adaptive trilateral filter," Research Paper: International Journal of Recent Trends in Engineering,. Vol.1, No.1, May 2009.

[9] S.Peng and L.Lucke, "Multi level adaptive fuzzy filter for mixed noise removal," in Proc. IEEE Int. Symp. Circuits Systems, Seattle, WA Apr. 1995, vol.2, pp. 1524-1527.

[10] M. Elad, "On the origin of the bilateral filter And ways to improve it" IEEE Trans.Image Processing vol.11,pp.114-1151,Oct.,2002.

[11] R H.Chan, C.W.Ho, and M.Nikolova, "Salt and pepper noise removal by median-type noise detectors and detail preserving regularization," IEEE Trans. Image Process., vol. 14, no. 10, pp. 1479-1485,Oct. 2005.

[12] Geoffrine Judith.M. and N.Kumarasabapathy, "Study and analysis of impulse noise reduction filters, "Signal \& Image Processing : An International Journal (SIPIJ) Vol.2, No.1, March 2011.

[13] I. Pitas and A. N. Venetsanopoulos, Nonlinear Digital Filters: Principles and Applications. Norwell, MA:Kluwer, 1990.

[14] E. Abreu, M. Lightstone, S. Mitra, and K. Arakawa, "A new efficient approach for the removal of impulse noise from highly corrupted images," IEEE Trans. Image Processing, vol. 5, pp. 1012-1025, June 1996.

[15] Pankaj Kumar Sa, Ratnakar DasH and Banshidhar Majhi," Second order difference based detection and Directional weighted median filter for removal of random valued impulsive noise," Fourth IEEE International Conference on Industrial and Information Systemsf 28-31 December 2009, University of Peradeniya, SriLanka. 